Published in Kattan, M (ed.), Encyclopedia of Medical Decision Making. Sage 2009.

\title{
EVALUATING CONSEQUENCES
}

Decisions in medical contexts have immediate and obvious consequences in terms of health and sometimes death or survival. Medical decisions also have less obvious and less immediate consequences, including effects on the long-term physical and mental well-being of patients, their families and of care-givers, as well as on the distribution of scarce medical resources. Some of these consequences are hard to measure and estimate. Even harder, perhaps, is the determination of the relative value of different consequences. How should consequences be evaluated? How do uncertainties and biases affect our evaluations of consequences? What influence should our evaluations of consequences have on our actions? These questions are all philosophical in nature.

\section{Consequences and value}

To evaluate something is most basically to determine its value, or to determine its effect on that which has value. The positive value of health may be taken as a given in medical decision making. Sometimes, however, it is not clear what concrete outcomes contain more health. Will a patient in chronic pain be more healthy taking opiates that reduce her mental abilities and may create dependency, or will she be more healthy without opiates but with more pain? Will an elderly patient with myeloma enjoy better health after treatment with cytostatics that pacify the disease but weaken the immune system, or will her health be better without the treatment? Depending on the details of the case, the answers to these questions are far from obvious, showing that the concept of health is complex and will sometimes stand in need of specification.

Health may be defined biomedically as the absence of disease and infirmity. This is the common definition in medical practice, though seldom explicitly stated. Alternatively, health may be defined biopsychosocially, which is common in theoretical contexts. The 1946 constitution of the World Health Organization states that health is "a state of complete physical, mental and social well-being”. Several recent definitions aim to avoid the somewhat utopian character of the WHO definition and to shift focus from outcome to opportunity, by defining health in terms of potential or ability rather than well-being. 
Quantitative measurements of health have increasingly been made in terms of QALYs - quality adjusted life years, that is the number of person life years adjusted by a factor representing the quality of the person's life. Like health, quality of life may be defined biomedically or biopsychosocially, and more or less broadly. What will be said in the following about values in general and health in particular, holds equally for quality of life. Regardless of how exactly quality is defined, evaluating consequences in terms of QALYs incorporates a richer understanding of why we value life, as opposed to measuring only years of life of whatever quality, or only death or survival. A strategy of QALY maximization has the further advantage of allowing quantitative comparisons of different alternatives, such as treatment programs, but has the disadvantage that other values may be disregarded, such as equity and autonomy.

Like any value, the value of health may be final and/or instrumental. Health is obviously instrumental to other values such as happiness and achievement. In other words, we need health in order to promote or protect these other values. In addition, however, health may also be of final value - of value in itself, independently of its impact on other values. Whether or not health has final value becomes important in conflict cases, where it must be balanced against other values. If, for example, health, defined biomedically, is important only because of its instrumental contribution to the higher value of happiness, a healthy life without happiness has no value. This conclusion may have direct relevance for important medical decisions concerning life and death, including the issue of euthanasia.

Values may be subjective or objective. That the value of health is subjective would mean that health is of value only to the extent that the individual patient considers it to be of value, or to the extent that she desires it. That the value is objective, on the other hand, would mean that health may be of value despite the fact that the patient does not subjectively value it. That a value is objective does not mean that it is insensitive to individual preferences, since objective values depend on individual preferences indirectly. Even if happiness, for example, is objectively valuable, what makes people happy depends on their preferences. Similarly, even if health is objectively valuable, what makes people healthy will depend on their physical constitution and individual character, including preferences. Whether values are subjective or objective naturally affects how we should treat each other in medical and other contexts. 
Beyond the somewhat related values of health, quality of life, well-being and happiness, autonomy is arguably the main value relevant for medical decision making. This value is institutionalized through the practice of informed consent, but may be affected also in other ways. For example, addictions may be considered to decrease autonomy and so treatment of addiction may promote autonomy. Further values of possible relevance include dignity, equity, personal relationships, and perfection or excellence. Dignity may be relevant to hospice care and other care of dying patients, equity to any decision affecting the distribution of scarce medical resources, relationships to how families are treated and to decisions affecting the patients' potential to uphold personal relationships after treatment, and perfection may be relevant to neonatal screening and genetic and medical enhancement.

Which things have objective value, if any, is a fundamental philosophical question and opinions and theories diverge. Lacking agreement, we may look to social value as determined by willingness to pay or stated preference, or to politically, hopefully democratically, determined values, or to expert judgment, or to our own judgment. Again, opinions and theories diverge. What we can say, rather trivially, is that consequences of decisions should be evaluated in terms of those things that are determined to have value.

If more than one value is affected by a decision, as seems likely for most medical decisions, we must determine how these values relate to each other. Most fundamentally, values may or may not be commensurable. If the value of health and the value of autonomy are incommensurable, we cannot weigh one against the other and so must make decisions that affect both values without guidance from such weighing. If the values are commensurable, they may be more or less open to comparison. At one end of the spectrum, we may know only that a little health is less important than a lot of autonomy, but not know how to compare much of each or little of each. At the other end of the spectrum, any amount of each value may be represented by a number and the values aggregated in multiattribute utility analysis. The very different character of some values may make them seem incommensurable, while the need to make decisions that affect more than one value forces us to compare them, or at least to act as if we had compared them. 


\section{Uncertainties and biases}

In evaluating consequences, we are inescapably faced with a number of uncertainties and biases. It is widely recognized that we do not even know if established medical practice on the whole efficiently promotes best outcomes (though the growing field of outcomes research aims to address that question). The uncertainty is naturally greatest for consequences of decision not yet made. We often do not know what consequences will follow from alternative courses of action. In evaluating possible future consequences, these uncertainties can to some extent be handled by decision theoretical methods. If we are uncertain what consequences will follow, we may at least know, or be able to estimate approximately, the probabilities of different possible outcomes, each with a set of consequences. Given these probabilities, we may estimate the expected value of different alternatives. To a large extent, however, uncertainty about the future must simply be accepted as a fact of life.

Uncertainty does not only pertain to future consequences, but also to the value of consequences, future as well as past and present. Even if we know that we value health and we know the consequences of a certain decision, we might not know to what extent those consequences further our values. This may be because we are not certain how exactly our values should be specified, or because we are not certain how much the concrete consequences contribute to our values, however thoroughly specified. For example, if health is defined in terms of ability we may not know to what extent successful treatment of radical mastectomy will contribute to this value. A person's overall ability depends partly on her attitudes, and patients may react differently to this medical procedure even when the physical outcome is the same.

Uncertainty about the value of consequences is increased by different sorts of biases. We tend to exaggerate the impact of certain things and belittle the impact of others. Some biases concerning our own well-being have been rather straightforwardly proven by psychological research. For example, we tend to overvalue variation in our consumption in the sense that we opt beforehand for variation but regret this once we get it. Other biases are harder to prove. For example and importantly, we value good things in the near future higher than similarly good things in the more distant future, and the reverse for bad things. This means for example that the social value of QALYs in the distant future is much lower than the social value of the same number and quality of 
life years in the near future. Whether this is an irrational bias that should be compensated for or an indication of our true values is a matter of controversy.

Uncertainties about consequences introduce another level of value - it requires us to determine how much we value certainty. A program of maximization of expected QALYs presumes that one QALY for sure is as good as a one in two chance of two QALYs. This is not so if we are risk-averse, that is if we value goods that we are certain to get higher than goods we may or may not get, even when the expected value is the same. In fact, people tend to be risk-averse. However, this may be considered an irrational bias.

\section{Consequences and principles}

In bioethics, principles are often understood as non-rigid rules and recommendations that must be interpreted in concrete cases with a large dose of moral judgment. Such principles are essentially statements of what has value, with the add-on that we have a duty to promote or protect that value. The question of which bioethical principles there are and how they should be understood corresponds to the question of what values there are and how they should be understood. Whether one prefers duty-talk or value-talk depends on whether one finds duty or value to be the more fundamental moral category. This is another matter on which opinions or sentiments diverge.

There are other kinds of principles, however, that do not as closely resemble values, but that rather regulate the evaluation of consequences. Some of these principles are rules of thumb, stating that for practical reasons such as time constraint and limited information and information processing capacity, we should restrict our evaluation of consequences in different ways. A rule that the most severely injured should be treated first may be such a rule. It is not a deep moral truth that the most severely injured deserves first treatment, but in most cases the rule is fair and efficient and reasonably easy to follow without time-consuming judgment. That this is a rule of thumb rather than a fundamental principle is shown by our reactions to the hypothetical case where there are obvious reasons to diverge from the rule, for example that it is clear that the most severely injured will not benefit from quick treatment while others will. If diverging from the rule in such circumstances is morally unproblematic, then the rule is one of thumb. By contrast, while a moral principle may be overridden, this is not 
unproblematic but normally give cause for regret and may give rise to residual obligations.

Rules of thumb replace or restrict evaluations of consequences for practical reasons. Moral principles do so for moral reasons. There are essentially two sorts of moral principles. Action-focused principles, or side constraints, state that certain things must or may not be done, regardless of other consideration. Examples include general principles such as 'never lie' as well as specifically medical principles such as 'never force medical care on a patient against her explicit wish'. Reason-focused or valuefocused principles, by contrast, state that certain reasons or values should be disregarded in the molding of various consideration into an all things considered judgment of what should be done. An example is the principle that a patient's estimated future contribution to society should not influence our medical treatment of her.

Many principles are tied to our social and legal roles, for example as medical practitioners. These roles come with social expectations, rules and laws, which regulate how and to what extent we must and may consider certain consequences of our actions. If such role principles are motivated only by expedience, they may be seen as rules of thumb. However, if they become ingrained in the culture of a society, they acquire the status of moral principles. Even as rules of thumb, role principles are unusually rigid, because they are motivated by practical reasons on a collective or system level. While individual practitioners may on occasion have time and capacity to judge a case on its own merits, they may be obliged to follow rules nonetheless, because this makes for stability and transparence in the medical system as a whole. The rigidity of role principles should not be exaggerated, however. The social and legal frameworks rarely if ever determine in detail how we should act and think. Even in applying well defined rules, we need value judgments to guide our application of those rules to particular circumstances. Furthermore, as rational and moral beings we can always question the social and legal framework within which we live and work.

\section{A model for evaluating consequences}

The different aspects of evaluating consequences covered above may be captured in the following model. This somewhat novel model incorporates a series of not so novel considerations. The model does not describe how evaluations are performed in practice, but rather proscribes what steps should be taken in order that all the aspects of 
evaluation discussed above be considered. In other words, the model is not psychological, but philosophical. If implemented in practice, the steps of the model should not necessarily be taken in strict order. In particular, step 2, 3 and 4 may all require glancing ahead to subsequent steps.

1. Determine which things have value - i.e. which values there are. This includes deciding whether values are subjective or objective, and final or instrumental.

2. Determine the available alternatives.

3. Decide whether some alternative is demanded by principle. If so, act.

4. Decide whether some alternatives are forbidden by principle. If so, exclude them from further consideration. If only one alternative is not forbidden, act.

5. Estimate for each alternative the possible outcomes and the (approximate) probability of each outcome.

6. Estimate the consequences of each outcome in terms of each value, adjust for bias.

7. Decide whether the consideration of some values are forbidden by principle and if so disregard these values.

8. Estimate the expected consequence of each alternative in terms of each value.

Either:

9a. If values are commensurable: Estimate or decide the overall value of each alternative, act on the best alternative.

Or:

9b. If values are incommensurable: Act on the alternative with the most appealing or most acceptable mix of expected consequences. 
See also: Bioethics; Constructions of Values; Decision Rules; Expected Utility Theory; Health Outcomes Assessment; Moral Factors; Multiattribute Utility Theory; Outcomes Research; Protected Values; Quality-Adjusted Life Years (QALYs); Quality of WellBeing Scale; Risk Aversion; Values; Willingness to Pay

\section{Further reading}

Beauchamp, T.L., \& Childress, J.F. (2001). Principles of Biomedical Ethics, Fifth Edition. Oxford: Oxford University Press.

Bircher J. (2005). Towards a Dynamic Definition of Health and Disease. Medicine, Health Care and Philosophy, 8, 335-341.

Griffin, J. (1986). Well-being: Its Meaning, Measurement and Moral Importance. Oxford: Oxford University Press.

Hastie, R., \& Dawes, R.M. (2001). Rational Choice in an Uncertain World. Thousand Oaks, CA: Sage.

Kane, R.L. (2006). Understanding Health Care Outcomes Research. Sudbury, MA: Jones and Bartlett.

O’Neill O. (2001). Practical Principles \& Practical Judgment. Hastings Center Report, July-August, 15-23.

Raz, J. (2003). The Practice of Value. Oxford: Oxford University Press.

Savulescu, J., Gillon, R., Beauchamp, T.L., Macklin, R., Sommerville, A., Callahan, D., Campbell, A.V., Gardiner, P., \& Harris, J. (2003). Festschrift edition in honour of Raanan Gillon. Journal of Medical Ethics, 29, 265-312.

Schroeder, Mark. (2008). Value Theory. In E.N. Zalta (Ed.), The Stanford Encyclopedia of Philosophy (Spring 2008 Edition), URL = $<$ http://plato.stanford.edu/archives/spr2008/entries/value-theory/ >.

World Health Organization (1946). Constitution of the World Health Organization. In: Basic Documents. Geneva: World Health Organization. 